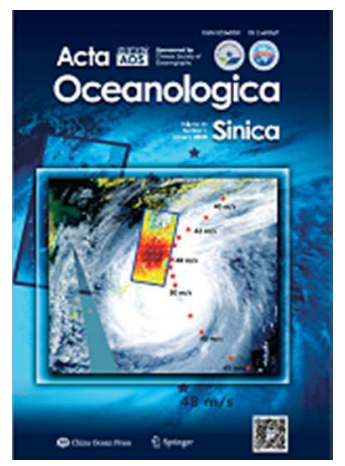

\title{
Comparison of linear and nonlinear extreme wave statistics
}

\begin{tabular}{|r|l|}
\hline Journal: & Acta Oceanologica Sinica \\
\hline Manuscript ID & AOS-2015-08-0204 \\
\hline Manuscript Type: & Original Article \\
\hline Keywords: & $\begin{array}{l}\text { extreme waves, Freak waves, probability of freak waves, direct } \\
\text { wave modeling }\end{array}$ \\
\hline Speciality: & Physical Oceanography \\
\hline \multicolumn{2}{|c}{} \\
\hline
\end{tabular}

SCHOLARONE

Manuscripts 


\section{Comparison of linear and nonlinear extreme wave statistics}

\section{Summary}

An extremely large ('freak') wave is a typical though rare phenomenon observed in the sea. Special theories (for example, the modulation instability theory) were developed to explain mechanics and appearance of freak waves as a result of nonlinear wave-wave interactions. In this paper it is demonstrated that the freak wave appearance can be also explained by superposition of linear modes with the realistic spectrum. The integral probability of trough-to-crest waves is calculated by two methods: the first one is based on the results of the numerical simulation of a wave field evolution performed with one-dimensional and two-dimensional nonlinear models. The second method is based on calculation of the same probability over the ensembles of wave fields constructed as a superposition of linear waves with random phases and the spectrum similar to that used in the nonlinear simulations. It is shown that the integral probabilities for nonlinear and linear cases are of the same order of values.

\section{Introduction}

Freak waves have always been a part of the marine folklore, but over the past 15-20 years have firmly found a place in the mainstream oceanography ${ }^{1}$. The meaning of the term 'freak' (or 'rogue') waves in science and life is quite different. According to the marine folklore, freak waves are described as 'monster' waves appearing like 'walls of water' (high crests) or 'holes in the sea' (deep troughs), or both. Another folklore variety of freak waves is 'three sisters' which can be interpreted as either a sequence of rogue waves or, more likely, an anomalous wave group.

In science 'freak' wave is defined as a wave whose trough-to-crest height exceeds twice the significant wave height $H_{s}$ ( $H_{s}$ is a characteristic mean wave height usually defined as a mean value of $1 / 3^{\text {rd }}$ of the highest waves). What seems remarkable is that the sea folklore provides a better description of the freak wave properties focusing on their shape rather than the height, while assuming, of course, that such waves are very big. The term 'vertical walls' definitely indicates a wave surge in front of the observer, i.e., the wave undergoes an active phase of breaking. However, the current scientific definition of the term 'freak wave' has a solid theoretical background since it is based on the fundamental properties of the adiabatic motion described by the Euler equations, i.e., these equations are self-similar, because, being transformed into a nondimensional form they do not contain a nondimensional parameter. Hence, the nondimensional equations describe the whole class of motions. Thus, for obtaining specific dimensional results, it is enough to multiply the solution by an appropriate length scale $L$. It can still be concluded that for the nondimensional equations the scientific definition of freak waves is justified.

For a long time scientists were not paying attention to the stories describing the events of 'monster' waves, reasonably presuming that big waves during strong storms do not suggest anything unusual. However, later it became clear that such waves do exist and look perhaps too big to be explained from the point of view of statistics. It was a starting point for developing many theoretical schemes (see an excellent review ${ }^{1}$ ). Some researchers explained generation of freak waves by external factors, such as an effect of wave energy focusing on the ocean currents 
of specific configurations. Obviously, such events deserve being investigated; however, the problem of freak waves appears to be much broader, since such waves have been detected in many areas of World Ocean with no special oceanic structures such as a surface current.

The most popular theory recently employed to explain the freak wave phenomenon is the so-called 'modulational instability theory' originally known as 'Benjamin-Feir (B.-F.) instability theory' ${ }^{2}$. The concept of this theory is quite transparent, i.e., the one-dimensional nonlinear wave in the presence of certain disturbances can produce additional modes arising in the vicinity of the main mode. Roughly speaking, the B.-F. theory explains redistribution of wave energy in a frequency (wavenumber) space up to the final homogenization of the initially discrete spectrum ${ }^{3}$. Most scientists believe that this mechanism can explain an abnormal growth of one wave mode. In case of the broad spectrum, typical for the wind-generated waves, such explanation is difficult to accept. First of all, it is unclear why one mode enjoys such preference and why this mode preserves its individuality in the course of its long development in a wave field with random phases. The original B.-F. results, as well as the and numerical investigations of B.-F. (Chalikov, 2007) showed that the period of new mode growth for the typical sea wave steepness exceeds hundreds or thousands of the carrying wave period. Thus, the freak wave should undergo a long course of development. Why do not interactions with other waves stop this growth, as if other waves do not exist?

The modulation instability theory of freak waves operates with such a poorly defined measure as the so-called BFI (Benjamin Feir Index) parameter calculated as a ratio of wave steepness $A K_{p}$ ( $A$ is a wave amplitude at spectral peak and $K_{p}$ is its wavenumber, both being dimensional), to the spectral bandwidth $\Delta K / K_{p} ; \Delta K$ being a measure of width of the spectrum estimated as a half-width at the half-maximum of spectrum ${ }^{4}$. Actually, the amplitude $A$ at spectral peak essentially depends on spectral resolution. The value of 'width' of spectrum is also uncertain since wave spectrum normally embraces a wide range of frequencies, so the value of BFI finally depends on somewhat arbitrary definitions.

Spectral analysis seems to be effective when it describes more or less uniform process like quadruplet interactions or energy input to waves, while it is rather pointless when applied to the analysis of extremely rare events represented by the single or multi-peak disturbances of a vast wave field. Such disturbances are evidently created locally in a physical space while they cannot manifest themselves in the wave spectrum which characterizes a large area.

No detailed data on time/space development of large waves are available, however, the results of the 2-D and 3-D mathematical modeling based on full equations show that the process of 'freaking' is very fast while the period of life of extreme waves is short. Such data do not prove an importance of the modulational instability theory for explanation of a freak wave phenomenon. The aim of this paper is to demonstrate new views on a freak wave problem.

\section{Numerical simulations}

The results presented below are obtained with the two-dimensional (2-D) and threedimensional (3-D) wave models. Both models are based on full nonlinear Euler equations in potential approximation. The 2-D model exploits conformal transformation of the coordinates, while the 3-D model uses the surface-following coordinate system and a direct solution of the three-dimensional equation for the velocity potential (see Appendix). The description of the 
models and demonstration of their accuracy are given $\mathrm{in}^{5,6,7}$. It is assumed here that the models are able to describe a real process with the sufficient accuracy. Both models were used in a quasi-adiabatic mode. It means that a small output of energy due to the flux of energy to subgrid domain is compensated by the input energy which is proportional to the wave spectrum. Below the non-dimensional variables with the following scales are used: length $L$ suggesting that $2 \pi L$ is a horizontal dimensional period; time $L^{1 / 2} g^{-1 / 2}$; and velocity potential $L^{3 / 2} g^{1 / 2}$ ( $g$ is the acceleration of gravity). The characteristics of the models are given in Table 1 ( $t_{p}$ is the time of integration expressed in a peak wave period).

Table 1. Characteristics of numerical models used for generation of the ensembles of surfaces.

\begin{tabular}{|c|c|c|c|c|c|c|}
\hline & $\begin{array}{c}\text { Spectral } \\
\text { resolution }\end{array}$ & $\begin{array}{c}\text { Grid } \\
\text { resolution }\end{array}$ & $\begin{array}{c}\text { Peak } \\
\text { wavenumber }\end{array}$ & Time step & $\begin{array}{c}\text { Number of } \\
\text { time steps }\end{array}$ & $\begin{array}{c}\text { Integration } \\
\text { time }\left(t_{p}\right)\end{array}$ \\
\hline $\begin{array}{c}3-\mathrm{D} \text { model } \\
1^{\text {st }} \text { run }\end{array}$ & $256 \times 128$ & $1024 \times 512$ & 16 & 0.005 & 200,000 & 636 \\
\hline $\begin{array}{c}3-\mathrm{D} \text { model } \\
2^{\text {nd }} \text { run }\end{array}$ & $512 \times 128$ & $2048 \times 512$ & 64 & 0.0025 & 100,000 & 318 \\
\hline 2-D model & 1000 & 4000 & 16 & 0.001 & $2,000,000$ & 1273 \\
\hline
\end{tabular}

The initial conditions in the models were assigned as a superposition of linear modes with random phases and amplitudes corresponding to the Pierson-Moskowitz spectrum ${ }^{8}$. In the 3-D model, a symmetric directional distribution was assigned for the energy-containing part of the spectrum proportional to $\cosh ^{n}(\theta)$ where $n=4$ was taken. The fields of surface elevation with time interval of $\delta t=1$ were recorded.

Analysis of the results was done in terms of the non-dimensional trough-to-crest wave height $H_{t c}$. The value $H_{t c}$ was defined as a difference between the maximum $H_{\max }$ and minimum $H_{\min }$ values of elevation normalized by the significant wave height:

$$
H_{t c}=H_{s}^{-1}\left(H_{\max }-H_{\min }\right)
$$

in the window with the size of $1.5 L_{p} \times 1.5 L_{p}$ where $L_{p}=2 \pi / k_{p}$ is the nondimensional peak wavelength. In 1-D case the window turns into a linear segment. The window was moved discreetly in both directions by $0.5 L_{p}$ step. Such window parameters were chosen in order to take into account all the range of possible values of $0 \leq H_{t c}$. Actually, $99 \%$ of $H_{t c}$ exceeds 1 . The shift being equal to $0.5 L_{p}$, but few values of $H_{t c}$ were sometimes taken more than once; anyway, it does not influence the statistics.

Such type of processing needs some explanation. Actually, we do not see any other ways to construct the true ensemble of $H_{t c}$ values; however, there is a big difference between the 1-D and 2-D cases. In 1-D case the maximum and minimum of the elevation fall on the same direction. In 2-D case these values can fall on different $y$-positions. That can be the main reason 
why the probability of large waves in 1-D case is normally lower than that in 2-D case. Naturally, with narrowing of the spectrum (i.e., at increase of power $n$ ) the 2-D distribution should approach the 1-D distribution. The probability of $H_{t c}$ wave height in 2-D case can also be calculated with a unidirected algorithm, when each vector of elevations along $x$ is processed as a result of the unidirected modeling. In this case the maximum and the nearest minimum of elevation coincide with a general direction of wave propagation. Note, however, that the 2-D algorithm (based on the square window) appears to be more practical.

A typical example of freak wave appearance is given in Fig. 1 where five consequent surfaces in a small fragment of the simulated domain are shown. The surfaces are reproduced
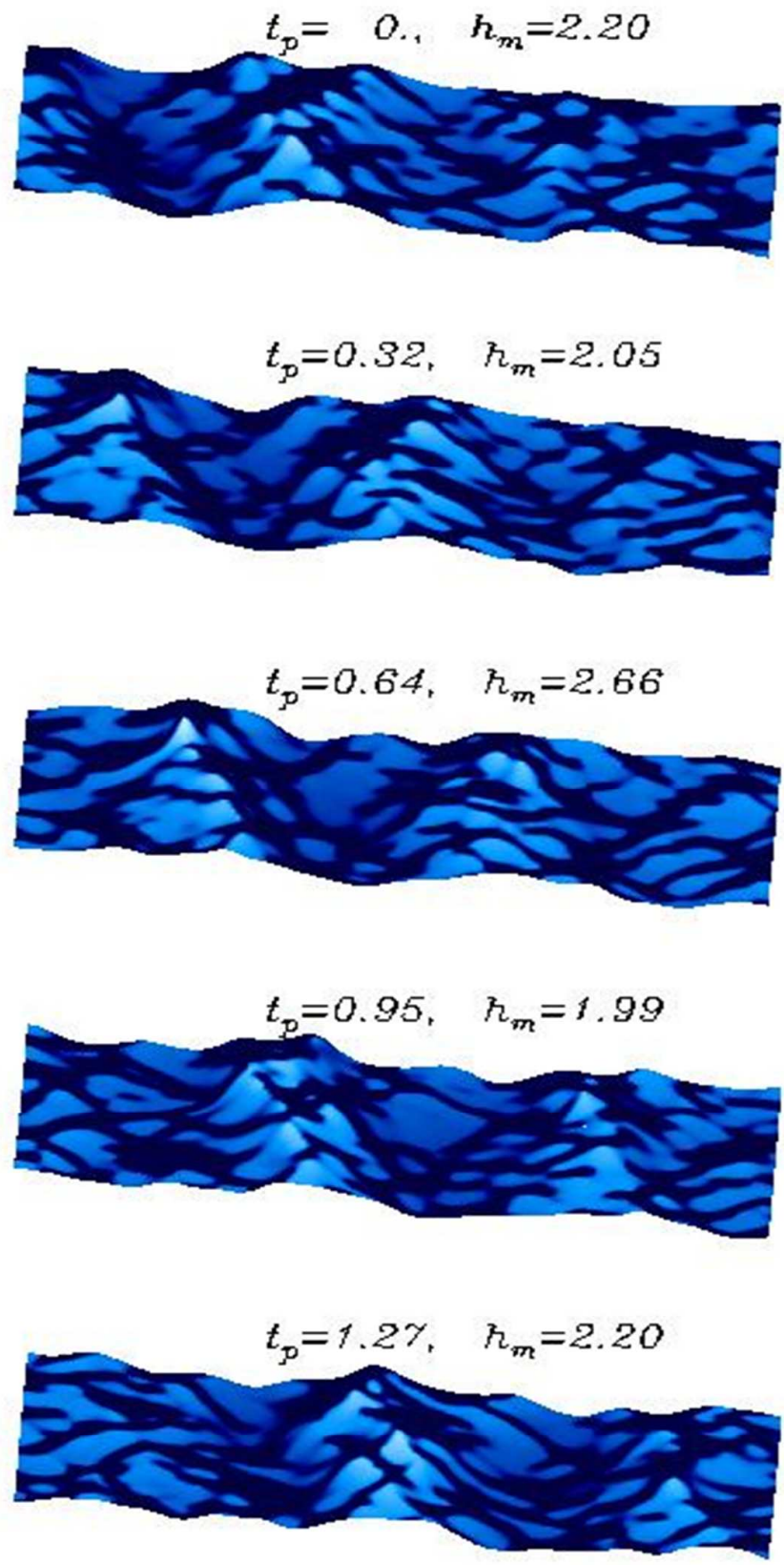
through interval of $0.32 t_{p}\left(t_{p} \approx 1.57\right.$ is the period of peak wave). As seen, the trough-to-crest height for a short period of the order of one peak wave period varies in the range of $1.90<H_{t c}<2.66$

Fig.1. Example of a short-term evolution of elevation. The same fragment of the surface is given for different moments separated by the interval of $0.32 t_{p}$. The largest normalized trough-to-crest wave height in the fragment is indicated.

(value $H_{t c}=2.66$ is the top value obtained in these simulations). In general, a shape of surface changes for such a short period significantly. Evolution of surface in Fig. 1 looks rather like an effect of dispersing superposition of different modes than the appearance of modulation instability.

A 'history' of extreme waves in domain $0<y<2$ for the period $600<t<640$ is shown in Fig. 2 where locations of large trough-tocrest waves (independent of their $x$ locations) are indicated: blue dots correspond to the values of $H_{t c}>1.7$ while red dots correspond to the values of $H_{t c}>2$. As seen, the dots are concentrated in groups, which proves their belonging to the same physical object. Many of the groups start from the blue dots and end also with the blue dots, while in the middle of the 
groups the red dots indicate freak waves. What is remarkable is that all these groups are short. It seems that freak waves arise suddenly with no prehistory, which also looks like as effect of superposition.

Let us consider local characteristics of wave fields, i.e. the average over 'jumping' with the step $0.5 L_{p}$ squared domain size of $1.5 L_{p} \times 1.5 L_{p}$

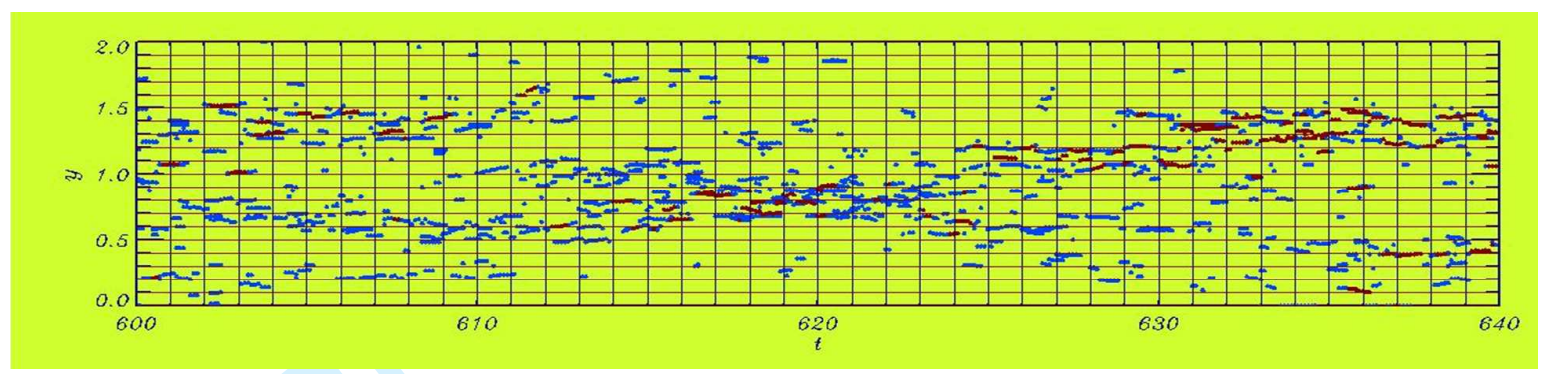

Fig.2. History of the extreme wave appearance in the coordinates $(t, y)$ for the period $600<t<640$ and the strip $0<y<2$. Blue dots correspond to the values $1.7<H_{t c}<2$, while red dots correspond to the values $H_{t c}>2$.

$$
\sigma=\left(\left(1.5 L_{p}\right)^{-2} \sum_{x} \sum_{y} \eta_{x}^{2}\right)^{1 / 2}
$$

Examples of the instantaneous field of rms steepness calculated in this moving window are given in Fig. 3. The upper panel refers to the data generated by the 3-D nonlinear model, while the bottom panel represents the data generated as a random superposition of linear waves.

$$
\eta=\sum_{k} \sum_{l} a_{k, l} \cos \left(k x+l y+\phi_{k, l}\right)
$$

where $k$ and $l$ are wavenumbers along the axes $x$ and $y, 0<\varphi_{k, l}<\pi$ is a random phase; amplitudes $a_{k, l}$ are calculated as follows:

$$
a_{k, l}=\left(2 S_{k, j} \Delta k \Delta l\right)^{1 / 2}
$$

( $\Delta k=\Delta l=1), S_{k, l}$ is the energy density spectrum.

Different sizes of dots in Fig. 3 characterize height of a freak wave. The field shown in the bottom panel of Fig. 3 looks as a typical member of the entire ensemble. Note that generating the fields with the nonlinear models took about two months of calculations, while the same size of ensemble of the fields calculated as a superposition of linear waves was generated for just 30 minutes. It can be expected that the wave fields obtained in so different a manner should be different as well. In particular, we expected that the averaged over windows wave steepness of a random superposition of linear modes should be more or less uniform. To our surprise, the pictures and animations generated for both cases demonstrated very similar features, i.e., the 
more or less uniform fields always contained small areas with a highly increased steepness. The locations of freak waves (marked as black dots in Fig. 3) always fell into these areas. It is difficult to understand what primary cause of such collocations is: either freak waves themselves increase local steepness or the increased local steepness is followed by appearance of freak waves (the 'chicken-and-egg' dilemma). Anyway, it is quite evident that the local steepness and height of an extreme wave in the window are connected with each other. This connection is demonstrated in Fig. 4 where an extreme trough-to-crest wave height is plotted as a function of the local steepness
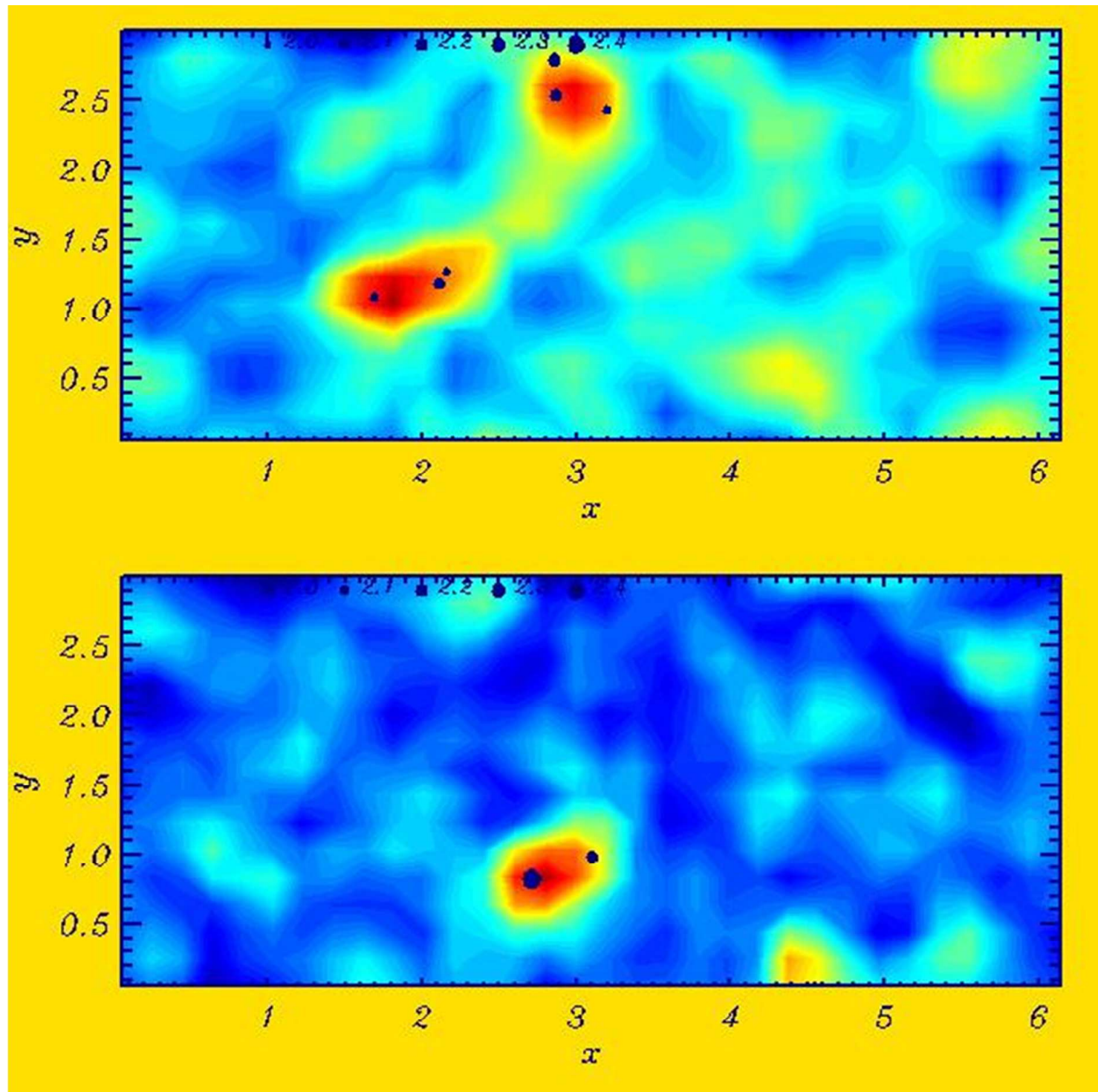

Fig.3. Examples of the instantaneous field of rms steepness calculated in the moving window (see description in the text). The upper panel refers to the data generated by the 3-D nonlinear model; the bottom panel represents the data generated as a random superposition of linear waves. The size of spots characterizes the height of freak waves (see legend on a top of picture).

Note that the high order moments such as skewness and kurtosis should demonstrate a much closer connection with extreme wave height than steepness. Of course, neither of all these characteristics can serve as predictors of freak waves (see Janssen, 2003), nor the freak waves 
can be predictors for the high order moments. The connection between the high order moments and height of waves is essentially local, which restricts applicability of the spectral analysis for a freak wave phenomenon.

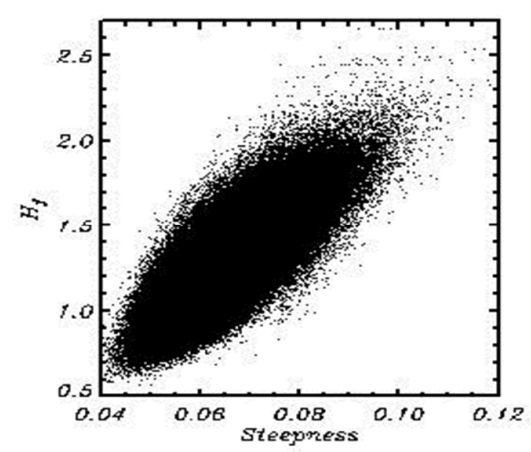

Fig.4.The rms steepness of elevation calculated over moving windows (see the text) vs. $H_{t c}$ found in each window.

Note that connection between the high order moments and height of extreme waves is well pronounced only for small domains. With extension of domain size this connection disappears (see Fig. 20 in Chalikov, 2007).
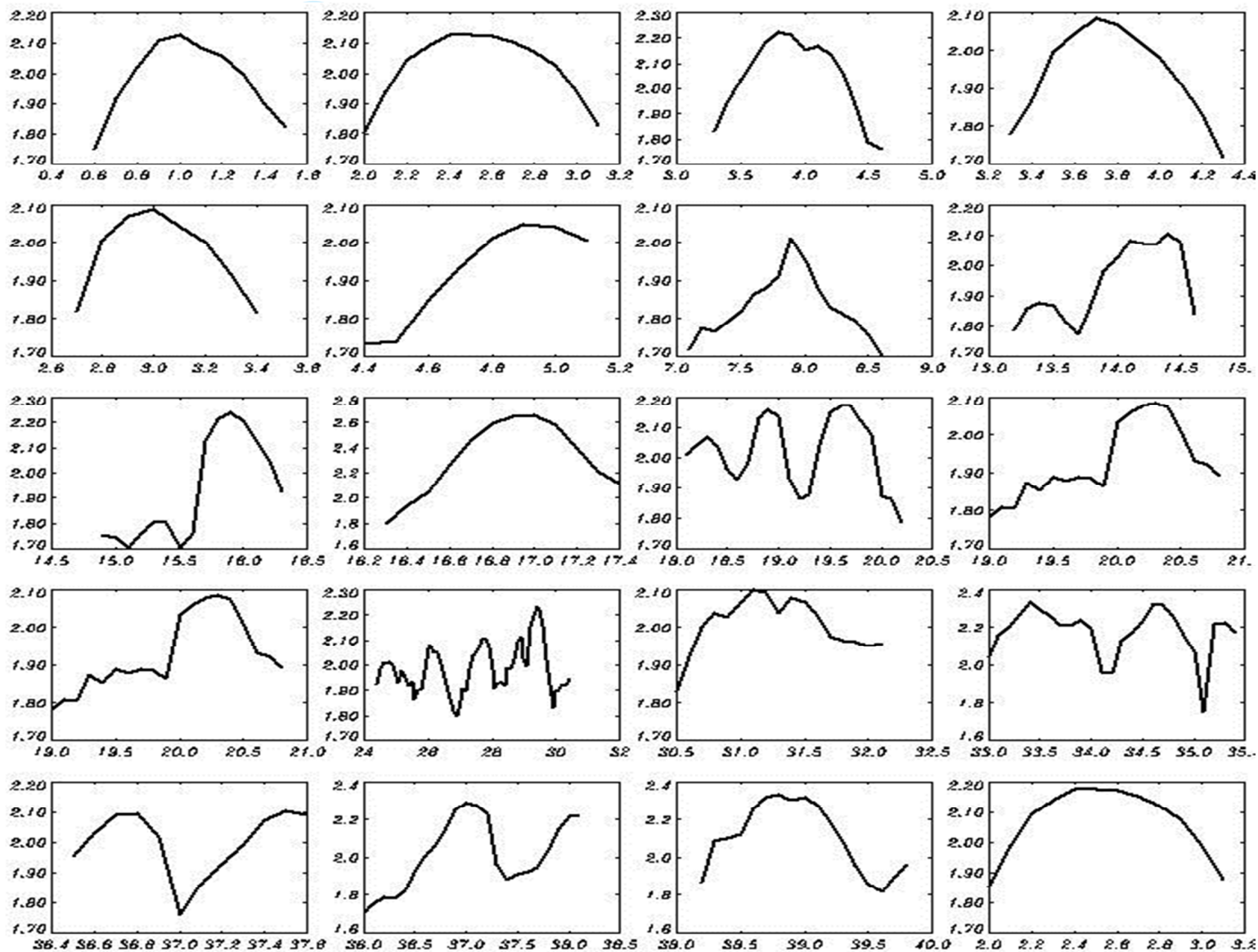

Fig. 5. Lagrangian evolution of trough-to-crest height $H_{t c}$. The horizontal line indicates value $H_{t c}=2$

A transient nature of freak waves demonstrated in Fig. 2 is well seen in the animations constructed from the pictures of Fig. 3. Both series demonstrate a very similar behavior. A transitory character of the extreme wave life can be also proved by Lagrangian tracing of the wave height represented in Fig. 5. More or less random choice of freak wave events 
demonstrates that a freak wave develops rapidly and exists over a very short time, i.e., approximately one peak wave period. Such behavior cannot be explained by the modulation instability theory; it looks rather like manifestation of a linear superposition of modes with different wave numbers and amplitudes.

The most convincing demonstration of a linear nature of extreme waves is given in Figs. 6 and 7 showing integral probability of the trough-to-crest height $H_{t c}$ of the waves. In Fig. 6 curves 1 and 3 are calculated using the results of a numerical simulation with the 3-D model (runs 1 and 3 refer to different resolutions); curves 2 and 4 are calculated over the same size ensembles of the fields represented as a superposition of linear waves. Surprisingly, the linear calculations give the same high values of large trough-to crest heights. Despite the fact that the data on the extremely high waves $\left(H_{t c}>2.5\right)$ are not stable, they do not allow us to state that probability of rare events is clearly different in all the cases considered. Note that the probability of $H_{c t}$ shown in Fig. 6 is considerably larger than that obtained with 1-D models (see, for example, Fig. 17 in Chalikov, 2009). It can be explained by a more general definition of freak waves in 2-D case (see Eq. 1).

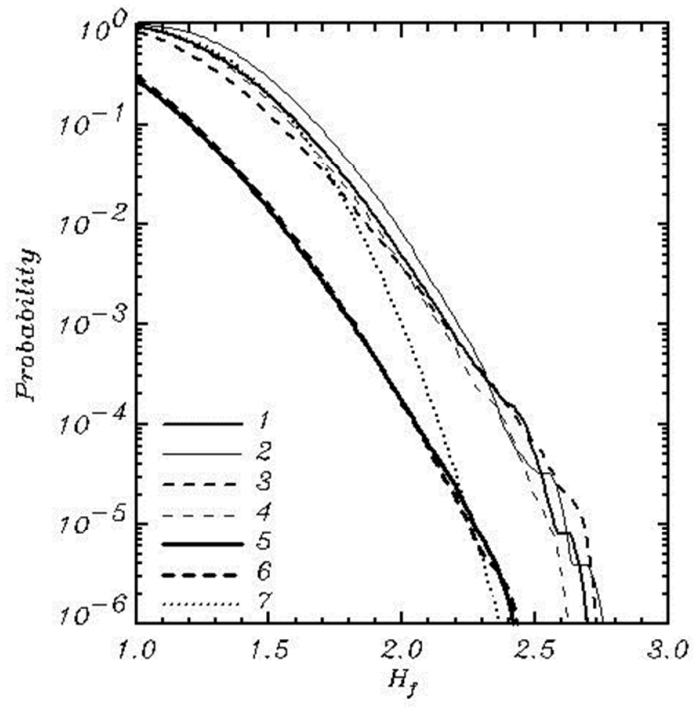

Fig. 6. Integral probability of trough-to-crest height $H_{t c}$. 1,2 - resolution $256 \times 128,3,4$ - resolution

$512 \times 128 ; 1,3$ - full 3-D nonlinear model; 2,4 - 2-D superposition of linear modes; 5,6 - 1-D treatment of $H_{t c}: 5$ - full nonlinear 3-D model; 6 -Superposition of 2-D linear modes; 7 - Raley distribution.

One-dimensional treatment of $H_{c t}$ (when square matrix turns into 1-D vector directed along the wave propagation) gives the probability smaller by more than one decimal order as compared to the previous algorithm. Remarkably, the probability obtained over the similar ensemble of the linear fields, actually coincides with the nonlinear results (curves 5 and 6). The curve 7 represents the Raley distribution calculated by the following relation:

$$
R=\exp \left(-\frac{\left(H_{t c}-\bar{H}_{t c}\right)^{2}}{2 \sigma_{t c}^{2}}\right),
$$

where $\bar{H}_{t c}=1.31$ and $\sigma_{t c}=0.22$ are the mean value and variance of $H_{t c}$ obtained by averaging over the data used for calculation of the probabilities 1,2,3 and 4, as described above. 
Fig.7. Integral probability of trough-to-crest height $H_{t c}$ for different angular spreadings: $1-P=4 ; 2-P=8$; 3- $P=16 ; 4-P=64 ; 5-P=256 ; 6-1-\mathrm{D}$ full nonlinear model; 7 - superposition of 1-D linear modes

The effect of narrowing of the window in $y$ direction is quite similar to the narrowing of the wave spectrum (i.e. increasing $n$ ). Such effect is demonstrated in Fig. 7 where the curves calculated for different values of $n$ are represented. Curve 1 is the same as the curve 1 in Fig. 6. As seen, with narrowing of spectrum the probability converges to that obtained with

\section{Conclusion.}

A definition of freak wave is based on the concept of the trough-to crest wave height, which is reasonable from the practical point of view. A natural wave field usually looks quite chaotic as a superposition of many dispersing modes which, in addition, are not conservative due to the fast reversible interactions. In our opinion, the only reasonable way to detect the instantaneous value of the trough-to crest height is detection of a maximum difference of elevations in the prescribed window. Since freak waves should be most likely associated with spectral peak, it is reasonable to choose the window with the size of the order of peak wave length and even somewhat bigger than that - for elimination of uncertainty of real wave length. Our experience shows that a size of domain should be of the order of $1.5 L_{p}$. In this case we do not take into account all possible extremes (because the maximum and minimum can sometimes be at a distance exceeding $1.5 L_{p}$ ), but the same structures can be taken twice. Such rare events happen sometimes; however, their influence on the statistics is quite insignificant.

In a one-dimensional wave field such a trough-to crest height definition gives quite definite results. However, in a two-dimensional wave field some uncertainty arises because the positions of maxima and minima can be shifted with respect to the direction of wave propagation. The simplest way to avoid uncertainty is to give a definition of the trough-to-crest height as a difference between the maximum and minimum along the direction coinciding with that of peak wave propagation. Such treatment of freak wave does not seem to be quite adequate, as the wave power depends on a full range of elevation.

The main result of the current investigation is comparison of the extreme wave statistics generated by the full nonlinear models and the statistics obtained over the ensemble of surfaces generated as a superposition of linear modes. In both cases the integral energy is the same, and the spectra of the surfaces are similar. The results obtained in this study are as follows:

1. Freak wave is a transient phenomenon; it develops and disappears approximately over the peak wave period. 
2. The wave fields generated as a superposition of linear modes with random phases show the properties very similar to those of the wave fields generated by $1-\mathrm{D}$ and $3-\mathrm{D}$ nonlinear models:

a. Both methods of generation demonstrate high probability of freak waves.

b. Integral probability of large waves for nonlinear and linear waves is roughly the same.

c. 2-D treatment of freak waves results in a significantly higher probability of freak waves than 1-D treatment or 1-D nonlinear simulations.

d. Probability of freak waves decreases with narrowing of the spectrum and approaches the probability obtained with 1-D models and 1-D superposition of linear modes.

Appendix.

\section{Numerical solution of three-dimensional fully nonlinear potential periodic waves}

Let us introduce the non-stationary surface-following non-orthogonal coordinate system

$$
\xi=x, \quad \vartheta=y, \quad \zeta=z-\eta(\xi, \vartheta, \tau), \quad \tau=t
$$

where $(x, y, z)$ are the Cartesian coordinates system, $t$ is time, $\eta(x, y, t)=\eta(\xi, \vartheta, \tau)$ is a moving periodic wave surface given by the 2-D Fourier series.

The 3-D equations of potential waves in the system of coordinates (6) at $\zeta<0$ take the following form:

$$
\begin{aligned}
& \eta_{\tau}=-\eta_{\xi} \varphi_{\xi}-\eta_{\vartheta} \varphi_{\vartheta}+\left(1+\eta_{\xi}^{2}+\eta_{\vartheta}^{2}\right) \Phi_{\varsigma}, \\
& \varphi_{\tau}=-\frac{1}{2}\left(\varphi_{\xi}^{2}+\varphi_{\vartheta}^{2}-\left(1+\eta_{\xi}^{2}+\eta_{\vartheta}^{2}\right) \Phi_{\zeta}^{2}\right)-\eta-p, \\
& \Phi_{\xi \xi}+\Phi_{\vartheta \vartheta}+\Phi_{\zeta \zeta}=\Upsilon(\Phi),
\end{aligned}
$$

where $\Phi$ is a three-dimensional velocity potential; $p$ is the external pressure; $\varphi$ is a value of $\Phi$ at surface $\zeta=0$ while $\Upsilon($ ) is the operator:

$$
\Upsilon()=2 \eta_{\xi}()_{\xi \zeta}+2 \eta_{\vartheta}()_{\vartheta \zeta}+\left(\eta_{\xi \xi}+\eta_{\vartheta \vartheta}\right)()_{\zeta}-\left(\eta_{\xi}^{2}+\eta_{\vartheta}^{2}\right)()_{\zeta \zeta}
$$

Equations (A2) and (A3) are written at the free surface whose position in the surfacefollowing coordinate system is fixed at $\zeta=0$. These equations (formally look as 2-dimensional; however, they include the vertical derivative of the potential $\Phi_{\zeta}$ which should be derived from the elliptical equation (A4) with the following boundary conditions:

$$
\Phi(\zeta=0)=\varphi, \quad \frac{\partial \Phi}{\partial \zeta}(\zeta \rightarrow-\infty)=0
$$


The second condition (A6) in the numerical scheme is replaced by the condition at the finite depth $\frac{\partial \Phi}{\partial \zeta}(\zeta=H)=0$ where depth $H$ should be large enough to be considered as infinitely large.

The potential is represented as a sum of two components, i.e. the analytic ('linear') one $\bar{\Phi},(\bar{\varphi}=\bar{\Phi}(\xi, \vartheta, 0))$ and an arbitrary non-linear component $\tilde{\Phi},(\tilde{\varphi}=\tilde{\Phi}(\xi, \vartheta, 0))^{1}$ :

$$
\varphi=\bar{\varphi}+\tilde{\varphi}, \quad \Phi=\bar{\Phi}+\tilde{\Phi}
$$

The analytic component $\bar{\Phi}$ satisfies the Laplace equation:

$$
\bar{\Phi}_{\xi \xi}+\bar{\Phi}_{\vartheta \vartheta}+\bar{\Phi}_{\zeta \zeta}=0
$$

with the known solution:

$$
\bar{\Phi}(\xi, \vartheta, \zeta)=\sum_{k, l} \bar{\varphi}_{k, l} \exp (|k| \zeta) \Theta_{k, l}
$$

( $\bar{\varphi}_{k, l}$ are the Fourier coefficients of the surface analytical potential $\bar{\varphi}$ at $z=0$ ). The solution satisfies the boundary conditions:

$$
\begin{aligned}
& \varsigma=0: \quad \bar{\Phi}=\bar{\varphi} \\
& \varsigma \rightarrow-\infty: \quad \tilde{\Phi}_{\zeta} \rightarrow 0
\end{aligned}
$$

The nonlinear component satisfies the equation:

$$
\tilde{\Phi}_{\xi \xi}+\tilde{\Phi}_{\vartheta \vartheta}+\tilde{\Phi}_{\zeta \zeta}=\Upsilon(\tilde{\Phi})+\Upsilon(\bar{\Phi})
$$

Eq. (20) is solved with the boundary conditions:

$$
\begin{aligned}
& \varsigma=0: \quad \tilde{\Phi}=0 \\
& \varsigma \rightarrow-\infty: \quad \tilde{\Phi}_{\zeta} \rightarrow 0
\end{aligned}
$$

Derivatives of the linear component $\bar{\Phi}$ are calculated directly with the use of (A9). The scheme combines the 2-D Fourier transform method in the 'horizontal surfaces' and the secondorder finite-difference approximation on stretched staggered grid defined by the relation $\Delta \zeta_{j+1}=\chi \Delta \zeta_{j}(\Delta \zeta$ is vertical step, and $j=1$ at the surface). The stretched grid provides increase of accuracy of approximation for the exponentially decaying modes. Values of the

\footnotetext{
${ }^{1}$ Note that the term 'linear' is conventional, since this component is also influenced by the non-linearity due to curvature of the surface.
} 
stretching coefficient $\chi$ lie within the interval 1.10-1.20. Finite-difference second-order approximation of Eq. (A11) on a non-uniform vertical grid is straightforward. The details of numerical scheme as well as results of simulation are given by Chalikov et al, (2014)

\section{References}

1. Kharif, C., Pelinovsky, E., Slunyaev, A. Rogue Waves in the Ocean. Advances in geophysical and environmental Mechanics and Mathematics. 2009, DOI 1007/978/-3540-88419-4

2. Benjamin, T.B., J. E. Feir:, 1967: The Disintegration of Wave Trains in Deep Water. $J$. Fluid. Mech., 1967, 27, 417-430

3. Chalikov, D. 2007: Simulation of Benjamin-Feir instability and its consequences. Physics of Fluid, 2007, 19, 016602-15

4. Onorato, M., Osborne, A., Serio, M., Bertone, S., Freak wave in random oceanic sea states. Physical Review Letters 2001, 86 (25), 5831-5834.

5. Chalikov D. D., Sheinin, Direct Modeling of One-dimensional Nonlinear Potential Waves. Nonlinear Ocean Waves, ed. W. Perrie, Advances in Fluid Mechanics, 1998, 17, 207- 258

6. Chalikov D., D. Sheinin, Modeling of Extreme Waves Based on Equations of Potential Flow with a Free Surface. Journ. Comp. Phys., 2005, 210, 247-273

7. Chalikov, D., Babanin AV. Numerical Modeling of Three-Dimensional Fully Nonlinear Potential Periodic Waves. Ocean dynamics., 2014, 64, 10, 1469-1486

8. Pierson, W. J. and Moscowitz, L. A proposed spectral form for fully developed wind seas based on the similarity theory of S A Kitaigorodskii, Journal of Geophys. Res., 1964, 69 (24), 5181-5190

9. Chalikov D. Freak waves: their occurrence and probability. Phys of Fluid, 2009, 21, 076602; doi:10.1063/1.3175713

10. Janssen, P., Nonlinear four-wave interaction and freak waves. J. Phys. Oceanogr., 2003, 33, 2001-2018

\section{Acknowledgements}

The authors thank Mrs. O. Chalikova for her assistance in preparation of the manuscript. The work was supported by RFBR, grant \#14-05-00422 and Australian Research Council, Discovery grants DP1093349 and DP130100227.

\section{Figures captions}

Fig.1. Example of a short-term evolution of elevation. The same fragment of the surface is given for a different moments, separated by the interval $0.32 t_{p}$. The largest normalized trough-to-crest wave height in the fragment is indicated. 
Fig.2. History of extreme wave appearance in coordinates $(t, y)$ for the period $600<t<640$ and the strip $0<y<2$. Blue dots correspond to the values $1.7<H_{t c}<2$, and red dots correspond to the values $H_{t c}>2$.

Fig.3. Examples of the instantaneous field of rms steepness calculated in a moving window (see description of window in the text). The upper panel refers to the data generated by 3-D nonlinear model; the bottom panel represents the data generated as a random superposition of linear waves. The size of spots characterizes the height of freak waves (see legend).

Fig.4.The rms steepness of elevation calculated over the moving windows (see description of window in the text) vs. $H_{t c}$ found in that window.

Fig. 5. Lagrangian evolution of trough-to-crest height $H_{t c}$. The horizontal line indicates value $H_{t c}=2$

Fig. 6. Integral probability of trough-to-crest height $H_{t c}$. 1, 2 - resolution $256 \times 128,3,4-$ resolution $512 \times 128$; 1,3 - full 3-D nonlinear model; 2,4 - 2-D superposition of linear modes; 5,6 - 1-D treatment of $H_{t c}: 5$ - full nonlinear 3-D model; 6 -Superposition of 2-D linear modes; 7 - Raley distribution.

Fig.7. Integral probability of trough-to-crest height $H_{t c}$ for different angular spreadings: 1 $P=4 ; 2-P=8 ; 3-P=16 ; 4-P=64 ; 5-P=256 ; 6$ - 1-D full nonlinear model; 7 superposition of $1-\mathrm{D}$ linear modes 


\section{Comparison of linear and nonlinear extreme wave statistics}

\section{Summary}

An extremely large ('freak') wave is a typical though rare phenomenon observed in the sea. Special theories (for example, the modulation instability theory) were developed to explain mechanics and appearance of freak waves as a result of nonlinear wave-wave interactions. In this paper it is demonstrated that the freak wave appearance can be also explained by superposition of linear modes with the realistic spectrum. The integral probability of trough-to-crest waves is calculated by two methods: the first one is based on the results of the numerical simulation of a wave field evolution performed with one-dimensional and two-dimensional nonlinear models. The second method is based on calculation of the same probability over the ensembles of wave fields constructed as a superposition of linear waves with random phases and the spectrum similar to that used in the nonlinear simulations. It is shown that the integral probabilities for nonlinear and linear cases are of the same order of values.

\section{Introduction}

Freak waves have always been a part of the marine folklore, but over the past 15-20 years have firmly found a place in the mainstream oceanography ${ }^{1}$. The meaning of the term 'freak' (or 'rogue') waves in science and life is quite different. According to the marine folklore, freak waves are described as 'monster' waves appearing like 'walls of water' (high crests) or 'holes in the sea' (deep troughs), or both. Another folklore variety of freak waves is 'three sisters' which can be interpreted as either a sequence of rogue waves or, more likely, an anomalous wave group.

In science 'freak' wave is defined as a wave whose trough-to-crest height exceeds twice the significant wave height $H_{s}$ ( $H_{s}$ is a characteristic mean wave height usually defined as a mean value of $1 / 3^{\text {rd }}$ of the highest waves). What seems remarkable is that the sea folklore provides a better description of the freak wave properties focusing on their shape rather than the height, while assuming, of course, that such waves are very big. The term 'vertical walls' definitely indicates a wave surge in front of the observer, i.e., the wave undergoes an active phase of breaking. However, the current scientific definition of the term 'freak wave' has a solid theoretical background since it is based on the fundamental properties of the adiabatic motion described by the Euler equations, i.e., these equations are self-similar, because, being transformed into a nondimensional form they do not contain a nondimensional parameter. Hence, the nondimensional equations describe the whole class of motions. Thus, for obtaining specific dimensional results, it is enough to multiply the solution by an appropriate length scale $L$. It can still be concluded that for the nondimensional equations the scientific definition of freak waves is justified.

For a long time scientists were not paying attention to the stories describing the events of 'monster' waves, reasonably presuming that big waves during strong storms do not suggest anything unusual. However, later it became clear that such waves do exist and look perhaps too big to be explained from the point of view of statistics. It was a starting point for developing many theoretical schemes (see an excellent review ${ }^{1}$ ). Some researchers explained generation of freak waves by external factors, such as an effect of wave energy focusing on the ocean currents of specific configurations. Obviously, such events deserve being investigated; however, the 
problem of freak waves appears to be much broader, since such waves have been detected in many areas of World Ocean with no special oceanic structures such as a surface current.

The most popular theory recently employed to explain the freak wave phenomenon is the so-called 'modulational instability theory' originally known as 'Benjamin-Feir (B.-F.) instability theory ${ }^{2}$. The concept of this theory is quite transparent, i.e., the one-dimensional nonlinear wave in the presence of certain disturbances can produce additional modes arising in the vicinity of the main mode. Roughly speaking, the B.-F. theory explains redistribution of wave energy in a frequency (wavenumber) space up to the final homogenization of the initially discrete spectrum ${ }^{3}$. Most scientists believe that this mechanism can explain an abnormal growth of one wave mode. In case of the broad spectrum, typical for the wind-generated waves, such explanation is difficult to accept. First of all, it is unclear why one mode enjoys such preference and why this mode preserves its individuality in the course of its long development in a wave field with random phases. The original B.-F. results, as well as the and numerical investigations of B.-F. (Chalikov, 2007) showed that the period of new mode growth for the typical sea wave steepness exceeds hundreds or thousands of the carrying wave period. Thus, the freak wave should undergo a long course of development. Why do not interactions with other waves stop this growth, as if other waves do not exist?

The modulation instability theory of freak waves operates with such a poorly defined measure as the so-called BFI (Benjamin Feir Index) parameter calculated as a ratio of wave steepness $A K_{p}\left(A\right.$ is a wave amplitude at spectral peak and $K_{p}$ is its wavenumber, both being dimensional), to the spectral bandwidth $\Delta K / K_{p} ; \Delta K$ being a measure of width of the spectrum estimated as a half-width at the half-maximum of spectrum ${ }^{4}$. Actually, the amplitude $A$ at spectral peak essentially depends on spectral resolution. The value of 'width' of spectrum is also uncertain since wave spectrum normally embraces a wide range of frequencies, so the value of BFI finally depends on somewhat arbitrary definitions.

Spectral analysis seems to be effective when it describes more or less uniform process like quadruplet interactions or energy input to waves, while it is rather pointless when applied to the analysis of extremely rare events represented by the single or multi-peak disturbances of a vast wave field. Such disturbances are evidently created locally in a physical space while they cannot manifest themselves in the wave spectrum which characterizes a large area.

No detailed data on time/space development of large waves are available, however, the results of the 2-D and 3-D mathematical modeling based on full equations show that the process of 'freaking' is very fast while the period of life of extreme waves is short. Such data do not prove an importance of the modulational instability theory for explanation of a freak wave phenomenon. The aim of this paper is to demonstrate new views on a freak wave problem.

\section{Numerical simulations}

The results presented below are obtained with the two-dimensional (2-D) and threedimensional (3-D) wave models. Both models are based on full nonlinear Euler equations in potential approximation. The 2-D model exploits conformal transformation of the coordinates, while the 3-D model uses the surface-following coordinate system and a direct solution of the three-dimensional equation for the velocity potential (see Appendix). The description of the models and demonstration of their accuracy are given $i^{5,6,7}$. It is assumed here that the models 
are able to describe a real process with the sufficient accuracy. Both models were used in a quasi-adiabatic mode. It means that a small output of energy due to the flux of energy to subgrid domain is compensated by the input energy which is proportional to the wave spectrum. Below the non-dimensional variables with the following scales are used: length $L$ suggesting that $2 \pi L$ is a horizontal dimensional period; time $L^{1 / 2} g^{-1 / 2}$; and velocity potential $L^{3 / 2} g^{1 / 2}$ ( $g$ is the acceleration of gravity). The characteristics of the models are given in Table 1 ( $t_{p}$ is the time of integration expressed in a peak wave period).

Table 1. Characteristics of numerical models used for generation of the ensembles of surfaces.

\begin{tabular}{|l|l|l|l|l|l|l|}
\hline & $\begin{array}{l}\text { Spectral } \\
\text { resolution }\end{array}$ & $\begin{array}{l}\text { Grid } \\
\text { resolution }\end{array}$ & $\begin{array}{l}\text { Peak } \\
\text { wavenumber }\end{array}$ & Time step & $\begin{array}{l}\text { Number of } \\
\text { time steps }\end{array}$ & $\begin{array}{l}\text { Integration } \\
\text { time }\left(t_{p}\right)\end{array}$ \\
\hline $\begin{array}{l}3-\mathrm{D} \text { model } \\
1^{\text {st }} \text { run }\end{array}$ & $256 \times 128$ & $1024 \times 512$ & 16 & 0.005 & 200,000 & 636 \\
\hline $\begin{array}{l}3-\mathrm{D} \text { model } \\
2^{\text {nd }} \text { run }\end{array}$ & $512 \times 128$ & $2048 \times 512$ & 64 & 0.0025 & 100,000 & 318 \\
\hline 2-D model & 1000 & 4000 & 16 & 0.001 & $2,000,000$ & 1273 \\
\hline
\end{tabular}

The initial conditions in the models were assigned as a superposition of linear modes with random phases and amplitudes corresponding to the Pierson-Moskowitz spectrum ${ }^{8}$. In the 3-D model, a symmetric directional distribution was assigned for the energy-containing part of the spectrum proportional to $\cosh ^{n}(\theta)$ where $n=4$ was taken. The fields of surface elevation with time interval of $\delta t=1$ were recorded.

Analysis of the results was done in terms of the non-dimensional trough-to-crest wave height $H_{t c}$. The value $H_{t c}$ was defined as a difference between the maximum $H_{\max }$ and minimum $H_{\min }$ values of elevation normalized by the significant wave height:

$$
H_{t c}=H_{s}^{-1}\left(H_{\max }-H_{\min }\right)
$$

in the window with the size of $1.5 L_{p} \times 1.5 L_{p}$ where $L_{p}=2 \pi / k_{p}$ is the nondimensional peak wavelength. In 1-D case the window turns into a linear segment. The window was moved discreetly in both directions by $0.5 L_{p}$ step. Such window parameters were chosen in order to take into account all the range of possible values of $0 \leq H_{t c}$. Actually, 99\% of $H_{t c}$ exceeds 1 . The shift being equal to $0.5 L_{p}$, but few values of $H_{t c}$ were sometimes taken more than once; anyway, it does not influence the statistics.

Such type of processing needs some explanation. Actually, we do not see any other ways to construct the true ensemble of $H_{t c}$ values; however, there is a big difference between the 1-D and 2-D cases. In 1-D case the maximum and minimum of the elevation fall on the same direction. In 2-D case these values can fall on different $y$-positions. That can be the main reason why the probability of large waves in 1-D case is normally lower than that in 2-D case. Naturally, 

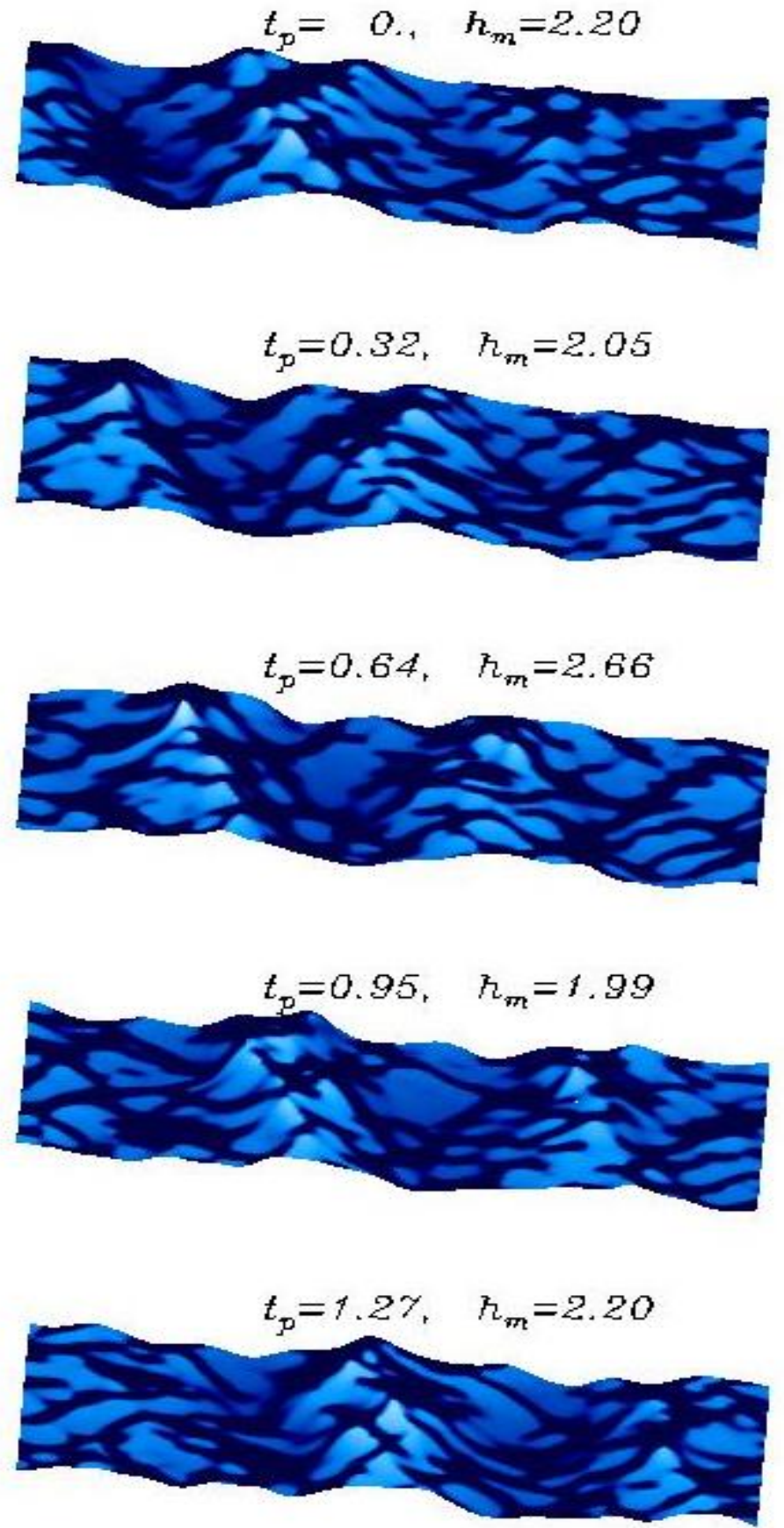
algorithm (based on the square window) appears to be more practical.

with narrowing of the spectrum (i.e., at increase of power $n$ ) the 2-D distribution should approach the 1-D distribution. The probability of $H_{t c}$ wave height in 2-D case can also be calculated with a unidirected algorithm, when each vector of elevations along $x$ is processed as a result of the unidirected modeling. In this case the maximum and the nearest minimum of elevation coincide with a general direction of wave propagation. Note, however, that the 2-D

A typical example of freak wave appearance is given in Fig. 1 where five consequent surfaces in a small fragment of the simulated domain are shown. The surfaces are reproduced through interval of $0.32 t_{p}\left(t_{p} \approx 1.57\right.$ is the period of peak wave). As seen, the trough-to-crest height for a short period of the order of one peak wave period varies in the range of $1.90<H_{t c}<2.66$

Fig.1. Example of a short-term evolution of elevation. The same fragment of the surface is given for different moments separated by the interval of $0.32 t_{p}$. The largest normalized trough-to-crest wave height in the fragment is indicated.

(value $H_{t c}=2.66$ is the top value obtained in these simulations). In general, a shape of surface changes for such a short period significantly. Evolution of surface in Fig. 1 looks rather like an effect of dispersing superposition of different modes than the appearance of modulation instability.

A 'history' of extreme waves in domain $0<y<2$ for the period $600<t<640$ is shown in Fig. 2 where locations of large trough-tocrest waves (independent of their $x$ locations) are indicated: blue dots correspond to the values of $H_{t c}>1.7$ while red dots correspond to the values of $H_{t c}>2$. As seen, the dots are concentrated in groups, which proves their belonging to the same physical object. Many of the groups start from the blue dots and end also with the blue dots, while in the middle of the groups the red dots indicate freak waves. What is remarkable is that all these groups are short. It 
167

168

169

170

171

172

173

174

175

176

177

178

179

180

181

182

183

184

185

186

187

188

189

190

191

192

193

194

195

196

197

198

199

seems that freak waves arise suddenly with no prehistory, which also looks like as effect of superposition.

Let us consider local characteristics of wave fields, i.e. the average over 'jumping' with the step $0.5 L_{p}$ squared domain size of $1.5 L_{p} \times 1.5 L_{p}$

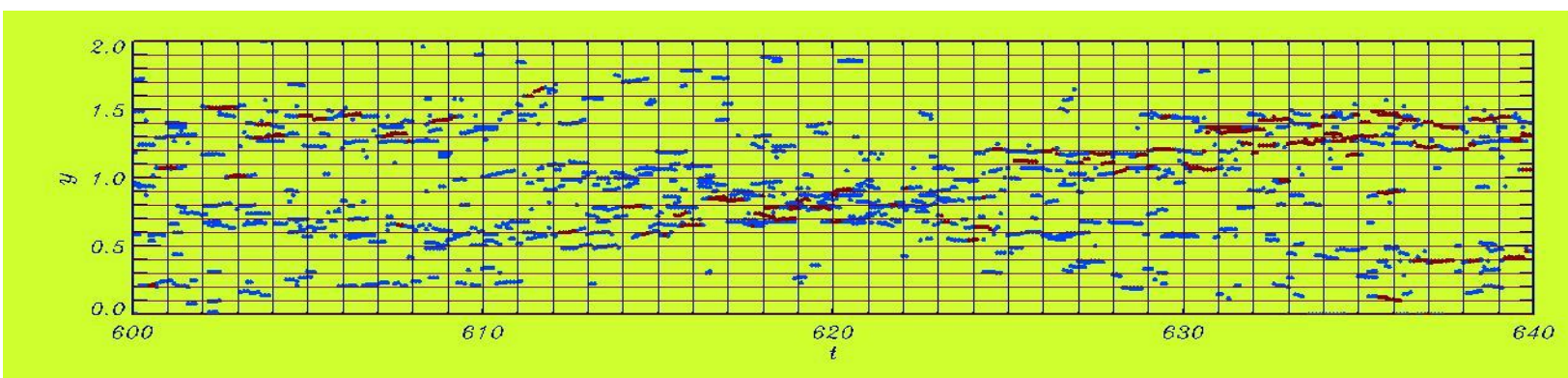

Fig.2. History of the extreme wave appearance in the coordinates $(t, y)$ for the period $600<t<640$ and the strip $0<y<2$. Blue dots correspond to the values $1.7<H_{t c}<2$, while red dots correspond to the values $H_{t c}>2$.

$$
\sigma=\left(\left(1.5 L_{p}\right)^{-2} \sum_{x} \sum_{y} \eta_{x}^{2}\right)^{1 / 2}
$$

Examples of the instantaneous field of rms steepness calculated in this moving window are given in Fig. 3. The upper panel refers to the data generated by the 3-D nonlinear model, while the bottom panel represents the data generated as a random superposition of linear waves.

$$
\eta=\sum_{k} \sum_{l} a_{k, l} \cos \left(k x+l y+\phi_{k, l}\right)
$$

where $k$ and $l$ are wavenumbers along the axes $x$ and $y, 0<\varphi_{k, l}<\pi$ is a random phase; amplitudes $a_{k, l}$ are calculated as follows:

$$
a_{k, l}=\left(2 S_{k, j} \Delta k \Delta l\right)^{1 / 2}
$$

( $\Delta k=\Delta l=1), S_{k, l}$ is the energy density spectrum.

Different sizes of dots in Fig. 3 characterize height of a freak wave. The field shown in the bottom panel of Fig. 3 looks as a typical member of the entire ensemble. Note that generating the fields with the nonlinear models took about two months of calculations, while the same size of ensemble of the fields calculated as a superposition of linear waves was generated for just 30 minutes. It can be expected that the wave fields obtained in so different a manner should be different as well. In particular, we expected that the averaged over windows wave steepness of a random superposition of linear modes should be more or less uniform. To our surprise, the pictures and animations generated for both cases demonstrated very similar features, i.e., the more or less uniform fields always contained small areas with a highly increased steepness. The 
locations of freak waves (marked as black dots in Fig. 3) always fell into these areas. It is difficult to understand what primary cause of such collocations is: either freak waves themselves increase local steepness or the increased local steepness is followed by appearance of freak waves (the 'chicken-and-egg' dilemma). Anyway, it is quite evident that the local steepness and height of an extreme wave in the window are connected with each other. This connection is demonstrated in Fig. 4 where an extreme trough-to-crest wave height is plotted as a function of the local steepness
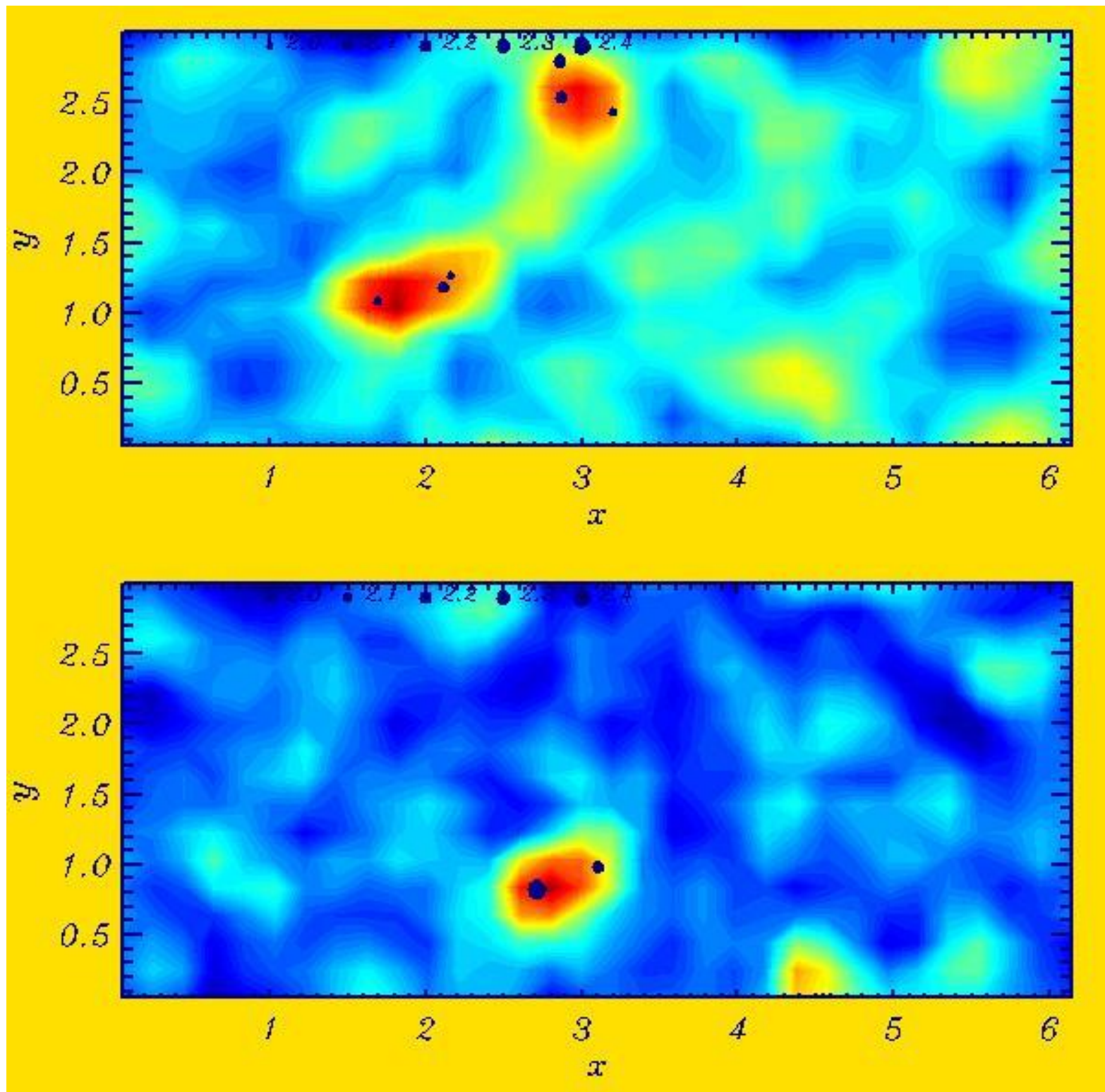

Fig.3. Examples of the instantaneous field of rms steepness calculated in the moving window (see description in the text). The upper panel refers to the data generated by the 3-D nonlinear model; the bottom panel represents the data generated as a random superposition of linear waves. The size of spots characterizes the height of freak waves (see legend on a top of picture).

Note that the high order moments such as skewness and kurtosis should demonstrate a much closer connection with extreme wave height than steepness. Of course, neither of all these characteristics can serve as predictors of freak waves (see Janssen, 2003), nor the freak waves can be predictors for the high order moments. The connection between the high order moments 
218 and height of waves is essentially local, which restricts applicability of the spectral analysis for a 219 freak wave phenomenon.

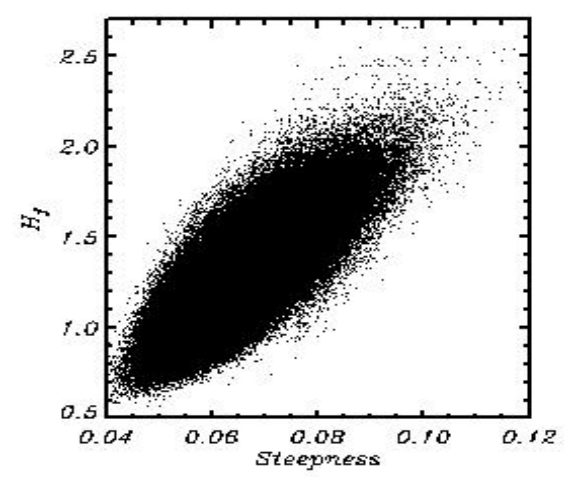

Fig.4.The rms steepness of elevation calculated over moving windows (see the text) vs. $H_{t c}$ found in each window.

Note that connection between the high order moments and height of extreme waves is well pronounced only for small domains. With extension of domain size this connection disappears (see Fig. 20 in Chalikov, 2007).
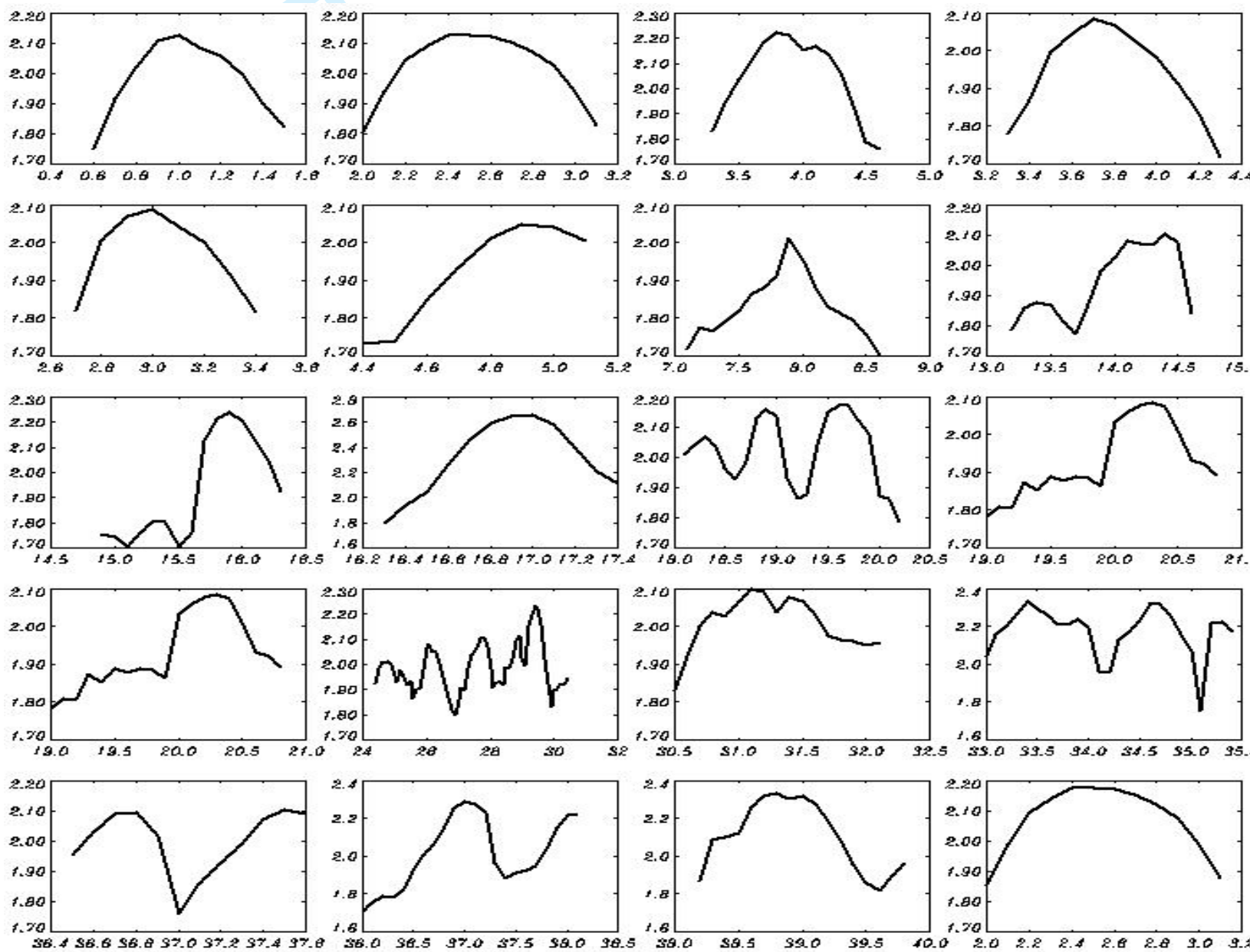

Fig. 5. Lagrangian evolution of trough-to-crest height $H_{t c}$. The horizontal line indicates value $H_{t c}=2$

232

233

234

235

236

237

A transient nature of freak waves demonstrated in Fig. 2 is well seen in the animations constructed from the pictures of Fig. 3. Both series demonstrate a very similar behavior. A transitory character of the extreme wave life can be also proved by Lagrangian tracing of the wave height represented in Fig. 5. More or less random choice of freak wave events demonstrates that a freak wave develops rapidly and exists over a very short time, i.e., 
238 approximately one peak wave period. Such behavior cannot be explained by the modulation 239 instability theory; it looks rather like manifestation of a linear superposition of modes with 240 different wave numbers and amplitudes.

241 The most convincing demonstration of a linear nature of extreme waves is given in Figs. 6 and 7 242 showing integral probability of the trough-to-crest height $H_{t c}$ of the waves. In Fig. 6 curves 1 and 2433 are calculated using the results of a numerical simulation with the 3-D model (runs 1 and 3 244 refer to different resolutions); curves 2 and 4 are calculated over the same size ensembles of the 245 fields represented as a superposition of linear waves. Surprisingly, the linear calculations give 246 the same high values of large trough-to crest heights. Despite the fact that the data on the 247 extremely high waves $\left(H_{t c}>2.5\right)$ are not stable, they do not allow us to state that probability of 248 rare events is clearly different in all the cases considered. Note that the probability of $H_{c t}$ shown 249 in Fig. 6 is considerably larger than that obtained with 1-D models (see, for example, Fig. 17 in 250 Chalikov, 2009). It can be explained by a more general definition of freak waves in 2-D case (see 251 Eq. 1).

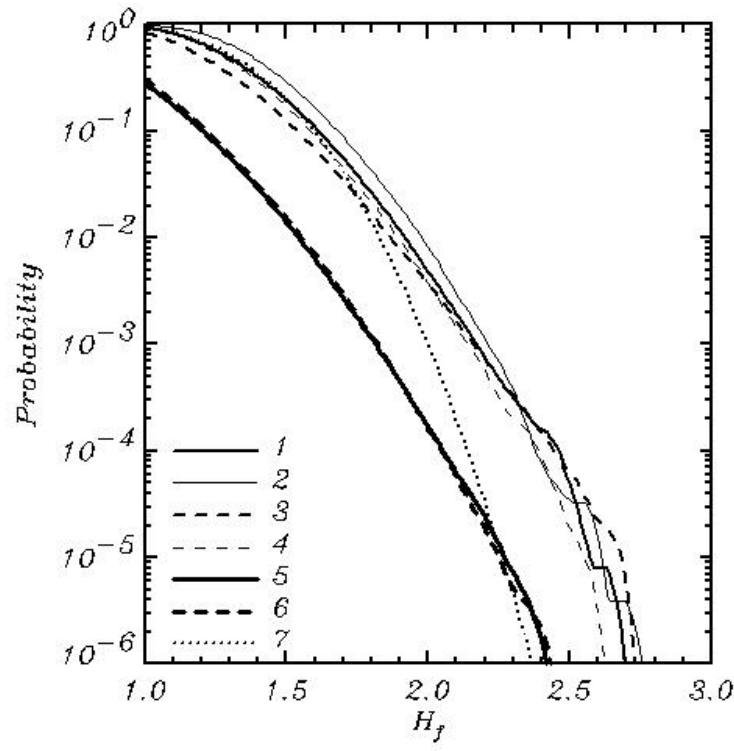

Fig. 6. Integral probability of trough-to-crest height $H_{t c}$. 1,2 - resolution $256 \times 128,3,4 \quad$ - resolution $512 \times 128 ; 1,3$ - full 3-D nonlinear model; 2,4 - 2-D superposition of linear modes; 5,6 - 1-D treatment of $H_{t c}: 5$ - full nonlinear 3-D model; 6 -Superposition of 2-D linear modes; 7 - Raley distribution.

One-dimensional treatment of $H_{c t}$ (when square matrix turns into $1-\mathrm{D}$ vector directed along the wave propagation) gives the probability smaller by more than one decimal

266

267

268 269

order as compared to the previous algorithm. Remarkably, the probability obtained over the similar ensemble of the linear fields, actually coincides with the nonlinear results (curves 5 and 6). The curve 7 represents the Raley distribution calculated by the following relation:

$$
R=\exp \left(-\frac{\left(H_{t c}-\bar{H}_{t c}\right)^{2}}{2 \sigma_{t c}^{2}}\right),
$$

where $\bar{H}_{t c}=1.31$ and $\sigma_{t c}=0.22$ are the mean value and variance of $H_{t c}$ obtained by averaging over the data used for calculation of the probabilities 1,2,3 and 4, as described above. 


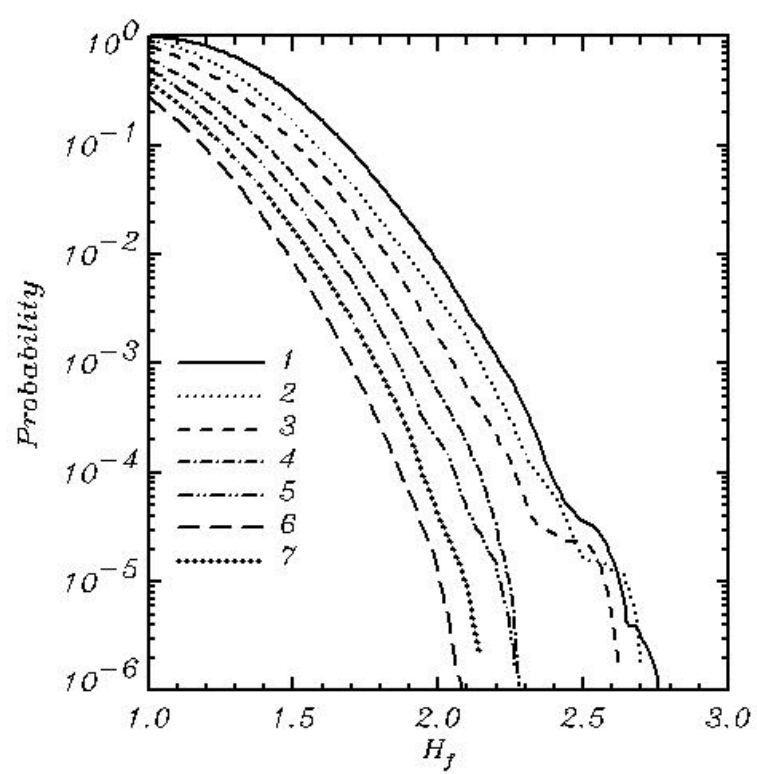

Fig.7. Integral probability of trough-to-crest height $H_{t c}$ for different angular spreadings: $1-P=4 ; 2-P=8$; $3-P=16 ; 4-P=64 ; 5-P=256 ; 6-1$-D full nonlinear model; 7 - superposition of 1-D linear modes

The effect of narrowing of the window in $y$ direction is quite similar to the narrowing of the wave spectrum (i.e. increasing $n$ ). Such effect is demonstrated in Fig. 7 where the curves calculated for different values of $n$ are represented. Curve 1 is the same as the curve 1 in Fig. 6. As seen, with narrowing of spectrum the probability converges to that obtained with

288 289 290

the 1-D model and the corresponding ensemble of 1-D linear surfaces.

\section{Conclusion.}

A definition of freak wave is based on the concept of the trough-to crest wave height, which is reasonable from the practical point of view. A natural wave field usually looks quite chaotic as a superposition of many dispersing modes which, in addition, are not conservative due to the fast reversible interactions. In our opinion, the only reasonable way to detect the instantaneous value of the trough-to crest height is detection of a maximum difference of elevations in the prescribed window. Since freak waves should be most likely associated with spectral peak, it is reasonable to choose the window with the size of the order of peak wave length and even somewhat bigger than that - for elimination of uncertainty of real wave length. Our experience shows that a size of domain should be of the order of $1.5 L_{p}$. In this case we do not take into account all possible extremes (because the maximum and minimum can sometimes be at a distance exceeding $1.5 L_{p}$ ), but the same structures can be taken twice. Such rare events happen sometimes; however, their influence on the statistics is quite insignificant.

In a one-dimensional wave field such a trough-to crest height definition gives quite definite results. However, in a two-dimensional wave field some uncertainty arises because the positions of maxima and minima can be shifted with respect to the direction of wave propagation. The simplest way to avoid uncertainty is to give a definition of the trough-to-crest height as a difference between the maximum and minimum along the direction coinciding with that of peak wave propagation. Such treatment of freak wave does not seem to be quite adequate, as the wave power depends on a full range of elevation.

The main result of the current investigation is comparison of the extreme wave statistics generated by the full nonlinear models and the statistics obtained over the ensemble of surfaces generated as a superposition of linear modes. In both cases the integral energy is the same, and the spectra of the surfaces are similar. The results obtained in this study are as follows:

1. Freak wave is a transient phenomenon; it develops and disappears approximately over the peak wave period. 
2. The wave fields generated as a superposition of linear modes with random phases show the properties very similar to those of the wave fields generated by $1-\mathrm{D}$ and $3-\mathrm{D}$ nonlinear models:

a. Both methods of generation demonstrate high probability of freak waves.

b. Integral probability of large waves for nonlinear and linear waves is roughly the same.

c. 2-D treatment of freak waves results in a significantly higher probability of freak waves than 1-D treatment or 1-D nonlinear simulations.

d. Probability of freak waves decreases with narrowing of the spectrum and approaches the probability obtained with 1-D models and 1-D superposition of linear modes.

\section{Appendix.}

\section{Numerical solution of three-dimensional fully nonlinear potential periodic waves}

Let us introduce the non-stationary surface-following non-orthogonal coordinate system

$$
\xi=x, \quad \vartheta=y, \quad \zeta=z-\eta(\xi, \vartheta, \tau), \quad \tau=t
$$

where $(x, y, z)$ are the Cartesian coordinates system, $t$ is time, $\eta(x, y, t)=\eta(\xi, \vartheta, \tau)$ is a moving periodic wave surface given by the 2-D Fourier series.

The 3-D equations of potential waves in the system of coordinates (6) at $\zeta<0$ take the following form:

$$
\begin{aligned}
& \eta_{\tau}=-\eta_{\xi} \varphi_{\xi}-\eta_{\vartheta} \varphi_{\vartheta}+\left(1+\eta_{\xi}^{2}+\eta_{\vartheta}^{2}\right) \Phi_{\varsigma}, \\
& \varphi_{\tau}=-\frac{1}{2}\left(\varphi_{\xi}^{2}+\varphi_{\vartheta}^{2}-\left(1+\eta_{\xi}^{2}+\eta_{\vartheta}^{2}\right) \Phi_{\zeta}^{2}\right)-\eta-p, \\
& \Phi_{\xi \xi}+\Phi_{\vartheta \vartheta}+\Phi_{\zeta \zeta}=\Upsilon(\Phi),
\end{aligned}
$$

where $\Phi$ is a three-dimensional velocity potential; $p$ is the external pressure; $\varphi$ is a value of $\Phi$ at surface $\zeta=0$ while $\Upsilon($ ) is the operator:

$$
\Upsilon()=2 \eta_{\xi}()_{\xi \zeta}+2 \eta_{\vartheta}()_{\vartheta \zeta}+\left(\eta_{\xi \xi}+\eta_{\vartheta \vartheta}\right)()_{\zeta}-\left(\eta_{\xi}^{2}+\eta_{\vartheta}^{2}\right)()_{\zeta \zeta}
$$

Equations (A2) and (A3) are written at the free surface whose position in the surfacefollowing coordinate system is fixed at $=0$. These equations (formally look as 2-dimensional; however, they include the vertical derivative of the potential $\Phi_{\zeta}$ which should be derived from the elliptical equation (A4) with the following boundary conditions:

$$
\Phi(\zeta=0)=\varphi, \quad \frac{\partial \Phi}{\partial \zeta}(\zeta \rightarrow-\infty)=0
$$


355 The second condition (A6) in the numerical scheme is replaced by the condition at the finite 356 depth $\frac{\partial \Phi}{\partial \zeta}(\zeta=H)=0$ where depth $H$ should be large enough to be considered as infinitely large.

357 The potential is represented as a sum of two components, i.e. the analytic ('linear') one 358 359

$$
=-\sim, \quad=-\sim
$$

The analytic component $\bar{\Phi}$ satisfies the Laplace equation:

$$
\bar{\Phi}_{\xi \xi}+\bar{\Phi}_{\vartheta \vartheta}+\bar{\Phi}_{\zeta \zeta}=0
$$

with the known solution:

$$
\bar{\Phi}(\xi, \vartheta, \zeta)=\sum_{k, l} \bar{\varphi}_{k, l} \exp (|k| \zeta) \Theta_{k, l},
$$

369

370

371

372

373

( $\bar{\varphi}_{k, l}$ are the Fourier coefficients of the surface analytical potential $\bar{\varphi}$ at $z=0$ ). The solution satisfies the boundary conditions:

$$
\begin{aligned}
& \varsigma=0: \quad \bar{\Phi}=\bar{\varphi} \\
& \varsigma \rightarrow-\infty: \quad \tilde{\Phi}_{\zeta} \rightarrow 0
\end{aligned}
$$

The nonlinear component satisfies the equation:

$$
\tilde{\Phi}_{\xi \xi}+\tilde{\Phi}_{\vartheta \vartheta}+\tilde{\Phi}_{\zeta \zeta}=\Upsilon(\tilde{\Phi})+\Upsilon(\bar{\Phi})
$$

Eq. (20) is solved with the boundary conditions:

$$
\begin{aligned}
& \varsigma=0: \quad \tilde{\Phi}=0 \\
& \varsigma \rightarrow-\infty: \quad \tilde{\Phi}_{\zeta} \rightarrow 0
\end{aligned}
$$

Derivatives of the linear component $\bar{\Phi}$ are calculated directly with the use of (A9). The scheme combines the 2-D Fourier transform method in the 'horizontal surfaces' and the secondorder finite-difference approximation on stretched staggered grid defined by the relation $\Delta \zeta_{j+1}=\chi \Delta \zeta_{j}(\Delta \zeta$ is vertical step, and $j=1$ at the surface). The stretched grid provides increase of accuracy of approximation for the exponentially decaying modes. Values of the stretching coefficient $\chi$ lie within the interval 1.10-1.20. Finite-difference second-order

\footnotetext{
${ }^{1}$ Note that the term 'linear' is conventional, since this component is also influenced by the non-linearity due to curvature of the surface.
} 
401

402

403

404

405

406

407

408

409

410

411

412

413

414

415

416

417

418

419

420

421

422

423

424

425

426

427

approximation of Eq. (A11) on a non-uniform vertical grid is straightforward. The details of numerical scheme as well as results of simulation are given by Chalikov et al, (2014)

\section{References}

1. Kharif, C., Pelinovsky, E., Slunyaev, A. Rogue Waves in the Ocean. Advances in geophysical and environmental Mechanics and Mathematics. 2009, DOI 1007/978/-3540-88419-4

2. Benjamin, T.B., J. E. Feir:, 1967: The Disintegration of Wave Trains in Deep Water. J. Fluid. Mech., 1967, 27, 417-430

3. Chalikov, D. 2007: Simulation of Benjamin-Feir instability and its consequences. Physics of Fluid, 2007, 19, 016602-15

4. Onorato, M., Osborne, A., Serio, M., Bertone, S., Freak wave in random oceanic sea states. Physical Review Letters 2001, 86 (25), 5831-5834.

5. Chalikov D. D., Sheinin, Direct Modeling of One-dimensional Nonlinear Potential Waves. Nonlinear Ocean Waves, ed. W. Perrie, Advances in Fluid Mechanics, 1998, 17, 207- 258

6. Chalikov D., D. Sheinin, Modeling of Extreme Waves Based on Equations of Potential Flow with a Free Surface. Journ. Comp. Phys., 2005, 210, 247-273

7. Chalikov, D., Babanin AV. Numerical Modeling of Three-Dimensional Fully Nonlinear Potential Periodic Waves. Ocean dynamics., 2014, 64, 10, 1469-1486

8. Pierson, W. J. and Moscowitz, L. A proposed spectral form for fully developed wind seas based on the similarity theory of S A Kitaigorodskii, Journal of Geophys. Res., 1964, 69 (24), 5181-5190

9. Chalikov D. Freak waves: their occurrence and probability. Phys of Fluid, 2009, 21, 076602; doi:10.1063/1.3175713

10. Janssen, P., Nonlinear four-wave interaction and freak waves. J. Phys. Oceanogr., 2003, 33, 2001-2018

\section{Acknowledgements}

The authors thank Mrs. O. Chalikova for her assistance in preparation of the manuscript. The work was supported by RFBR, grant \#14-05-00422 and Australian Research Council, Discovery grants DP1093349 and DP130100227.

\section{Figures captions}

Fig.1. Example of a short-term evolution of elevation. The same fragment of the surface is given for a different moments, separated by the interval $0.32 t_{p}$. The largest normalized trough-to-crest wave height in the fragment is indicated. 
428 Fig.2. History of extreme wave appearance in coordinates $(t, y)$ for the period $600<t<640$ 429 and the strip $0<y<2$. Blue dots correspond to the values $1.7<H_{t c}<2$, and red dots correspond 430 to the values $H_{t c}>2$.

431 Fig.3. Examples of the instantaneous field of rms steepness calculated in a moving window (see 432 description of window in the text). The upper panel refers to the data generated by 3-D nonlinear 433 model; the bottom panel represents the data generated as a random superposition of linear waves. 434 The size of spots characterizes the height of freak waves (see legend).

435 Fig.4.The rms steepness of elevation calculated over the moving windows (see description of 436 window in the text) vs. $H_{t c}$ found in that window.

437 Fig. 5. Lagrangian evolution of trough-to-crest height $H_{t c}$. The horizontal line indicates value $438 H_{t c}=2$

439 Fig. 6. Integral probability of trough-to-crest height $H_{t c}$. 1, 2 - resolution $256 \times 128,3,4$ 440 resolution $512 \times 128 ; 1,3$ - full 3-D nonlinear model; 2,4 - 2-D superposition of linear modes; 4415,6 - 1-D treatment of $H_{t c}: 5$ - full nonlinear 3-D model; 6 -Superposition of 2-D linear modes; 4427 - Raley distribution.

443 Fig.7. Integral probability of trough-to-crest height $H_{t c}$ for different angular spreadings: 1 $444 P=4 ; 2-P=8 ; 3-P=16 ; 4-P=64 ; 5-P=256 ; 6-1$-D full nonlinear model; 7 445 superposition of $1-D$ linear modes 